\title{
Dificultades en la lectura: percepción de los estudiantes que inician estudios universitarios. Caso Fundación Universitaria María Cano (FUMC), Sede Medellín
}

\author{
Reading Difficulties: Appraisal of the Students who \\ are Beginning their University Studies at FUMC \\ Case, Premise Medellin
}

\section{Mary Luz Osorio M.}

Fonoaudióloga

Magíster en Educación - Docencia

Fundación Universitaria María Cano,

FUMC

Medellín, Colombia

\author{
Ricardo León Sánchez A. \\ Ingeniero electricista \\ Magíster en Administración y \\ Planificación Educativa \\ Fundación Universitaria María Cano, \\ FUMC \\ Medellín, Colombia
}

Contacto con el autor:

Mary Luz Osorio M.

Calle 56 \# 41-90, Medellín, Antioquia

Medellín - Colombia

Tel: (00 574) 3217092227

Correo-e:

maryluzosoriomontoya@fumc.edu.co

Recibido: 04/06/2014

Aceptado: 20/07/2015

\begin{abstract}
RESUMEN
Los docentes universitarios se encuentran con estudiantes que presentan dificultades para leer y comprender los textos. La lectura es una estrategia para el aprendizaje significativo y para la comunicación. Los planteamientos que se realizan en este artículo están orientados por la investigación "Caracterización de dificultades en habilidades básicas del lenguaje: escuchar, hablar, leer y escribir en los estudiantes de primer semestre, año 2012-1, en la Fundación Universitaria María Cano (en adelante FUMC), sede Medellín", que tiene como objetivo identificar las dificultades en la habilidad básica del lenguaje: lectura. La investigación utiliza un enfoque cuantitativo con un método descriptivo; su diseño es no experimental, transversal y de campo. El análisis se realizó con el paquete estadístico Statgraphics Centurion XVII. Se evidenció que, desde la percepción de los estudiantes evaluados y según el análisis estadístico, se percibían dificultades en cada una de las variables independientes analizadas (Estado visual, Memoria, Atención, Motivación y Comprensión). Lo anterior puede afectar el rendimiento lector $y$, por ende, el desempeño académico de los estudiantes.
\end{abstract}

Palabras clave: lectura, estado visual, atención, motivación, memoria y comprensión.

\begin{abstract}
University professors have to deal with students who present difficulties in reading comprehension. Reading is a strategy for meaningful learning and communication. Information provided in the present article is based on the research entitled "Characterization of difficulties in basic language skills: listening, speaking, reading and writing on the freshmen in the first semester of 2012, at the Fundación Universitaria María Cano at Medellin. Objective of study is to identify the difficulties perceived in the basic language skill: reading, by the freshmen during the first semester in 2012. The present study was developed using a quantitative approach with a descriptive method. Research design is nonexperimental, cross-sectional and of field. For the statistical analysis, the software Statgraphics Centurion XVII was used. Difficulties in every independent variable were observed (visual state, memory, attention, motivation and comprehension). Freshmen of the first semester of 2012 at FUMC presented difficulties in all variables, affecting reading performance and thus academic performance.
\end{abstract}

Keywords: reading, vision, attention, motivation, memory, comprehension. 


\section{Introducción}

En la universidad la lectura es una actividad académica fundamental, una herramienta que enriquece los argumentos para establecer diálogos de saberes sin límites, al mismo tiempo es una estrategia de comunicación a través de la cual se establece un diálogo entre el emisor (quien a través del texto escrito comunica un mensaje) y el receptor (quien lo interpreta o decodifica). En este orden de ideas, se considera que si hay comprensión, se da retroalimentación y, por ende, un aprendizaje significativo. Al respecto, Zorrilla (2005) señala que entre el lector y el texto se establece una interacción donde la compresión se convierte en un proceso en el que el lector emplea las claves proporcionadas por el autor, en función de su propio conocimiento o experiencia previa, para inferir el significado que este pretende comunicar.

Tanto la codificación (organización del mensaje) como la decodificación (interpretación del mensaje) en el proceso lector pueden verse afectadas por factores internos y externos que inciden positiva o negativamente para que exista, por un lado, comunicación académica efectiva $y$, por el otro, aprendizaje significativo en los sujetos.

Es pertinente resaltar que en el proceso lector se ven implicadas las funciones cognitivas, las cuales determinan en parte el logro de los objetivos académicos; por ende, conocer esta realidad puede favorecer nuevas dinámicas de enseñanza y comunicación en las aulas. Asimismo, se considera que la lectura es una competencia que se desarrolla en los sujetos con el apoyo académico y familiar, los cuales son contextos que fortalecen significativamente los procesos lingüísticos y de enseñanza-aprendizaje; del mismo modo, estos permiten al sujeto acceder al conocimiento con autonomía y sentido crítico. Autores como Muter, Hulme, Snowling y Stevenson (citado por López y Guevara, 2008), definen la lectura como:

Una habilidad lingüística que, con raras excepciones, es aprendida solo después de que el niño ha adquirido un nivel de competencia considerable en el lenguaje oral; la falta de competencia lingüística se traduce frecuentemente en inhabilidades para el aprendizaje de la lectura (p. 60).

Lo que indica, como puede notarse, que hogar y escuela estimulan su aprendizaje.

Por otro lado, el proceso lector involucra diversos pasos, cada uno con un nivel de complejidad significativo donde el cerebro cumple un papel indispensable. El conocimiento de ello permite que los educadores actuales reflexionen acerca del funcionamiento cerebral y el mecanismo de comprensión de los estudiantes. Un dispositivo que permitió avanzar en el conocimiento del funcionamiento cerebral es la neuroimagen a través de la cual los especialistas centraron, inicialmente, el interés en el mapa anatómico de las funciones cerebrales. En tal sentido, León (2010) realizó una descripción de lo que ocurre específicamente con el lenguaje, ya que este tipo de mapas señala dos puntos importantes: a) el lenguaje no se limita a las áreas clásicas de Broca y Wernicke (de hecho, se introduce la mayor parte de áreas corticales de los dos hemisferios tanto como otras regiones subcorticales); y b) no existe una sola área del cerebro dedicada específicamente al lenguaje, sino 
que todas ellas también están implicadas en otras funciones no lingüísticas relacionadas con el control cognitivo, la memoria, la atención y la percepción o acción. Esta nueva visión establece una posibilidad educativa, puesto que involucra aspectos indispensables en el proceso lector, como lo son la comprensión y las inferencias.

En la lectura, el cerebro es el director de los resultados finales que se ejecutan durante el proceso de codificación y decodificación del mensaje escrito, lo cual se logra gracias a la mediación de los dispositivos básicos de aprendizaje y del lenguaje como función mental superior. Cada dispositivo o función, en forma independiente, realiza una acción compleja y organizada, desencadenando un efecto conjunto que compromete a todo el sistema cerebro-, el cual integra, asimila y acomoda esas acciones interdependientes para proyectar unas consecuencias que favorecen o afectan los objetivos del ejercicio lector.

A continuación se presentan y desarrollan conceptualmente los dispositivos y la función mental superior en el contexto de la lectura.

Sensopercepción. Es un dispositivo que contempla, dentro de sus posibilidades, la percepción visual. Mediante esta, los estímulos son seleccionados, discriminados e interpretados, dependiendo de las estructuras internas del sujeto que los percibe y las características externas de lo percibido. Bravo (2004) expresa cómo la enseñanza de la lectura no puede ser desvinculada del desarrollo del lenguaje y, en general, del desarrollo integral del sujeto, donde las destrezas perceptuales son esenciales en dicho aprendizaje. Este dispositivo se considera un proceso complejo por la relación con los otros procesos cognitivos. Cabe señalar que la capacidad del ojo humano para percibir los diversos estímulos le confiere al lector una herramienta importante para captar, discriminar y comprender las representaciones gráficas presentadas en los textos.

Otro de los dispositivos básicos de aprendizaje que participan en la lectura es la atención. Su importancia radica en el proceso selectivo que realiza, debido a la imposibilidad del sistema nervioso para procesar todo lo que recibe al mismo tiempo. De esta manera, la atención focaliza y concentra aquello que el sujeto desea, aunque la atención también puede ser involuntaria. Al respecto, Etchepareborda y Abad-Mas (2005) explican cómo funciona este dispositivo. Por una parte, lo hace en forma automática cuando orienta los sistemas sensoriales hacia las fuentes de información del entorno y, por otra, bajo control consciente en la medida en que, con frecuencia, se puede decidir cómo centrar la atención.

La motivación, en tanto, es otro dispositivo participante del proceso de lectura, definido como el conjunto de condiciones que inician, guían y mantienen unas actitudes abiertas por parte de los sujetos para el logro de los objetivos. En la lectura este mecanismo es indispensable, puesto que moviliza al lector a la acción y al descubrimiento de nuevas alternativas de interiorización del conocimiento. En tal sentido, Duque, Vera y Hernández (2010), comentan dos aspectos importantes de considerar: para comprender es conveniente tener en cuenta factores afectivos y motivacionales, y la comprensión se da como resultado de la interiorización, autorregulación y uso 
autónomo. Esto sugiere que el lector debe conocer los propios procesos implicados en la comprensión y cómo puede regularlos a través de distintas estrategias cognitivas y metacognitivas que deben ponerse en práctica antes, durante y después de la lectura.

No obstante, Ellery (2005), va más allá e introduce la reflexión metacognitiva, la cual hace a los alumnos conscientes de su propio proceso de aprendizaje y lo refuerza. Según la misma autora, los estudiantes son, entonces, capaces de elegir aquello que necesitan hacer para aprender y aplicar eficazmente estrategias para lograr un determinado nivel de éxito como lectores y escritores. La motivación, el apoyo familiar y las condiciones adecuadas permiten el desarrollo de diversas estrategias que benefician el proceso lector (Díaz, Martínez y Ávila, 2006).

En el proceso lector, la memoria es presentada como un dispositivo de aprendizaje que contiene todo acerca de lo que se piensa y se elabora en el presente; de ahí surge la expresión "memoria operativa". Logie (1995), sugiere que esta tiene tres componentes principales: el ejecutivo central, el lazo articulatorio, y la agenda viso-espacial.

De igual manera, Suárez, Moreno y Godoy (2010) confirman que uno de los aspectos cognitivos más relevantes es la memoria operativa. Al respecto, se ha comprobado que los alumnos mejoran este tipo de memoria cuando practican la lectura. Otros autores como Carpenter, Miyake y Just (1994), sostienen que las diferencias individuales en la capacidad de la memoria operativa afectan directamente la capacidad de lectura, en particular en el procesamiento de la sintaxis.

Autores como Etchepareborda y Abad-Mas (2005), definen la memoria como la capacidad de retener y evocar eventos del pasado, mediante procesos neurobiológicos de almacenamiento y recuperación de información básica en el aprendizaje y en el pensamiento. De tal forma, consideran que este sistema lo integran tres procesos: codificación de la información (donde se destaca la atención, concentración y el estado emocional del sujeto); almacenamiento de la información, que es un sistema complejo y dinámico que cambia con los hábitos a los que se expone el sujeto; y evocación o recuperación de la información.

El lenguaje es una función mental superior, Preilowski y Matute (2011) plantean al respecto que "el cerebro humano está estructurado para aprender por sí mismo, a comprender el lenguaje y a comunicar a través del habla" (p. 96). En relación con la lectoescritura, Borzone (citado por Villamizar, 2003) comenta:

Aprender a leer es una extensión natural de aprender a hablar, por lo que el aprendizaje de la lectoescritura se realiza sobre la base de los mismos procesos cognoscitivos y estrategias que el niño ha desarrollado al adquirir la lengua materna (p. 91).

Es necesario mencionar que, aunque la presente investigación se centra en la población juvenil, en el referente teórico se hizo alusión al desarrollo inicial del lenguaje, lo cual permitió inferir que muchas de las dificultades en lectoescritura que perciben los 
estudiantes de la muestra posiblemente existen desde la infancia y persisten en la edad adulta.

En relación con el planteamiento anterior, Defior (1993), gracias a sus trabajos investigativos, concluyó lo siguiente:

Creemos que la convergencia de las investigaciones en señalar una base lingüística en las dificultades de lectura y escritura, proporciona una guía para su aplicación tanto en los procesos de adquisición de estas habilidades como para la prevención, evaluación y tratamiento de los fracasos de aprendizaje (p. 8.).

Esta idea representa una oportunidad para fonoaudiólogos y maestros que trabajan en esta área, puesto que si logran identificar dificultades lingüísticas y de lectoescritura oportunamente, ejercerán acciones preventivas, de diagnóstico y de tratamiento, favoreciendo así las posibilidades de aprendizaje y el desarrollo intelectual en el ámbito académico.

En concordancia con lo anterior, es necesario definir conceptualmente dos procesos de la lectura en los que niños y jóvenes presentan dificultades. En primer lugar, se encuentra la decodificación, la cual se establece durante las primeras etapas del desarrollo del lenguaje (Shankweiler et al., 1999, citado por Muñoz y Schelstraete). Los autores afirman que la decodificación consiste en la aplicación eficiente del principio alfabético, explotando las regularidades entre las palabras y sus representaciones alfabéticas. De esta forma el sujeto, mediante este proceso, puede interpretar un vocabulario familiar e identificar nuevas palabras, lo que le permite fortalecer el léxico interno y consolidarse como un buen lector. En segundo lugar, se encuentra la comprensión, que en la lectura puede establecerse como un proceso de integración activa de información donde participan diferentes factores. Al leer, los sujetos establecen representaciones mentales del mensaje y de esta forma el lector reconoce grafemas, estructuras sintácticas e infiere significados de oraciones, párrafos y textos completos. Igualmente, la compresión implica las relaciones de cohesión del mensaje y las experiencias previas del lector (Abusamra, Cartoceti, Raiter y Ferreres, 2008).

A partir de las falencias observadas en el proceso lector de los estudiantes que ingresan a la universidad y el planteamiento teórico descrito en las líneas anteriores, se plantea como objetivo del presente trabajo identificar las dificultades en la habilidad básica del lenguaje (lectura) percibidas por los estudiantes de la FUMC, del primer semestre durante 2012. Lo anterior podría implicar un acercamiento inicial a la compleja problemática de las dificultades lectoras en los estudiantes universitarios.

\section{Método}

La investigación se desarrolla desde un enfoque cuantitativo con un método descriptivo y su diseño es no experimental, transversal y de campo. La unidad de análisis corresponde a estudiantes del primer semestre del año 2012 de la Fundación Universitaria María Cano, FUMC, Sede Medellín. El 
tamaño de esta población es de 382 individuos $^{1}$. El tipo de muestreo utilizado fue aleatorio simple y para la definición del tamaño de la muestra se tomó probabilidad de ocurrencia $p=0.5$, un margen de error $e=5.7 \%$ y un nivel de confianza del $95 \%$ $(z=1.96)$. Con los datos anteriores, se obtiene un tamaño de muestra $(n)$ de 167 estudiantes del primer semestre de 2012 de la FUMC. A continuación se describe el cálculo:

La fórmula utilizada para el cálculo del tamaño de la muestra $n$ en estos casos es:

$$
n=\frac{n_{0}}{1+\frac{n_{0}}{N}}
$$

Donde:

$$
n_{0}=\frac{z^{2} p(1-p)}{e^{2}}
$$

Con los datos descritos anteriormente obtenemos:

$$
n_{0}=\frac{1,96^{2} * 0,5 *(1-0,5)}{0,057^{2}}=295,6
$$

De lo cual resulta:

$$
n=\frac{295,6}{1+\frac{295,6}{382}}=166,64 \approx 167 \text { individuos }
$$

Una vez que se definió la muestra, se llevaron a cabo los siguientes pasos para el proceso de evaluación (aplicación de la prueba tamiz).

La autora, con un grupo de estudiantes próximos a terminar el programa de Fonoaudiología (en calidad de practicantes), seleccionó y organizó a los

${ }^{1}$ Datos suministrados por la oficina Admisiones y Registro de la FUMC. sujetos de la muestra, para lo cual se consideraron los diversos programas que existen en la FUMC y los horarios para determinar el momento de aplicación de la prueba. Con la autorización de los docentes responsables de cada grupo y el consentimiento informado diligenciado, se procedió a la aplicación del instrumento. Los estudiantes participantes estaban acompañados por los practicantes y asesores de práctica, con el objetivo de responder las inquietudes que surgieran en las respuestas dadas en el mismo instrumento.

Para la recolección de los datos, se utilizó como instrumento una prueba tamiz, la cual se ajustó a una escala Likert que contenía 39 preguntas, de las cuales se seleccionaron 18 . El estudiante elegía una respuesta a partir de las opciones presentadas en la siguiente tabla.

Tabla 1

Opciones de respuesta de la escala Likert

\begin{tabular}{l}
\hline Opción de respuesta \\
\hline Siempre \\
Muchas veces \\
A veces \\
Pocas veces \\
Nunca \\
\hline Fuente: Elaboración propia.
\end{tabular}

Para la medición, se cuantificó la escala Likert de respuestas utilizando los valores que se muestran a continuación.

Tabla 2

Valoración de la escala Likert de respuestas

\begin{tabular}{lc}
\hline Categoría (opción de respuesta) & Valor asignado \\
\hline Siempre & 5 \\
Muchas veces & 4 \\
A veces & 3 \\
Pocas veces & 2 \\
Nunca & 1 \\
\hline
\end{tabular}

Fuente: Elaboración propia. 
Estas opciones permitieron a los estudiantes seleccionar el nivel de percepción en relación con las cinco variables independientes: Estado visual, Memoria, Atención, Motivación y Comprensión.
Posteriormente se elaboró la Tabla 3, en la cual se muestra la operacionalización de las variables y las categorías e indicadores que permitieron recoger los datos para el análisis estadístico final.

Tabla 3

Operacionalización de variables

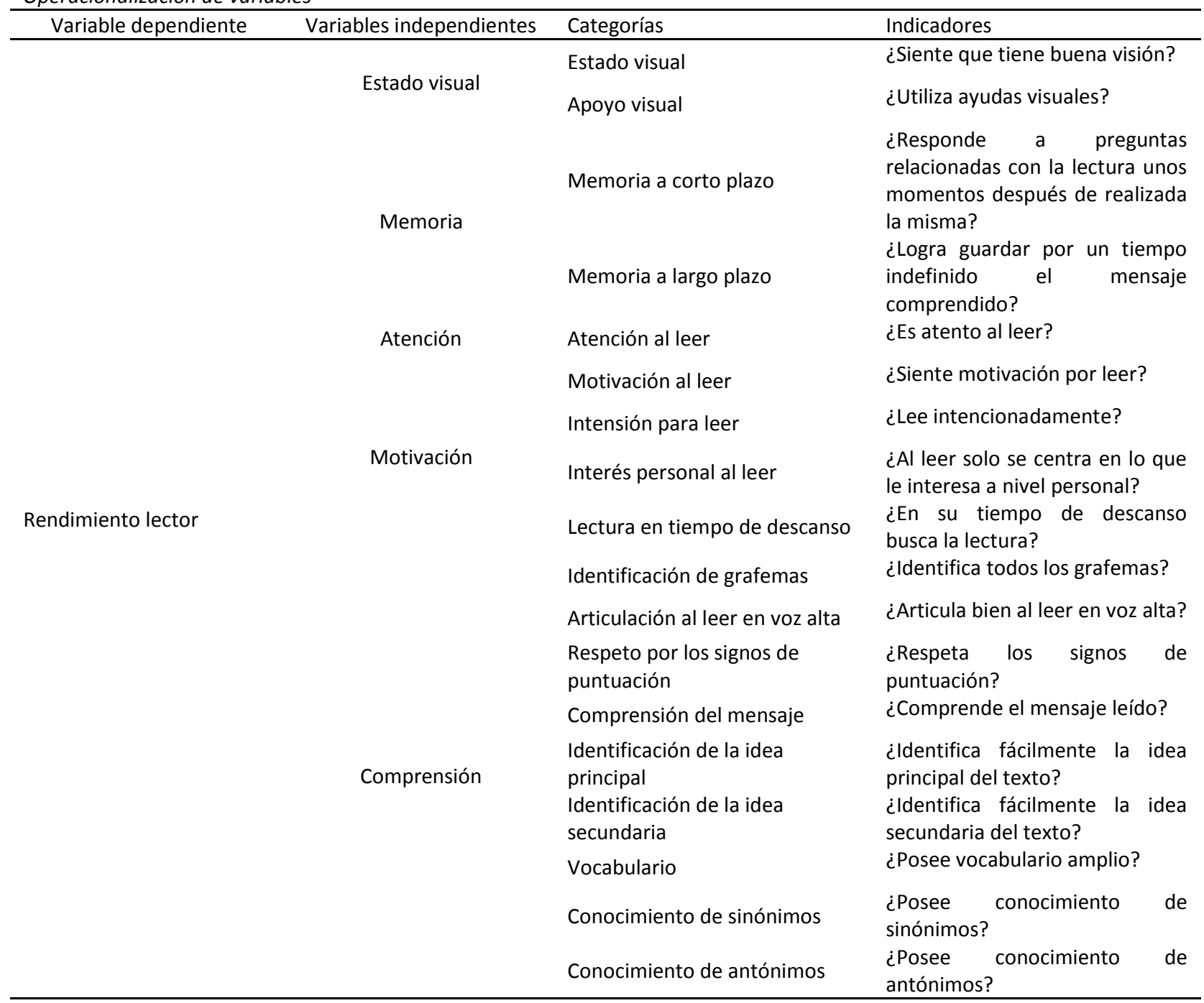

Fuente: Elaboración propia.

Con los resultados definidos en los pasos anteriores, se construyó la matriz de datos, y para el procesamiento de los mismos, se utilizó el paquete Statgraphics Centurion XVII el cual es un programa para gestionar y analizar valores estadísticos.

\section{Resultados}

Después de aplicar el instrumento de medición, se procedió a construir la matriz de datos mostrada en la Tabla 4, la cual contiene los resultados 
obtenidos para 15 de los 167 individuos (casos) seleccionados en la muestra. Por razones de espacio, esta matriz se presenta solo para algunas de las preguntas (indicadores) incluidas en la encuesta.

Tabla 4

Porción de la matriz de datos obtenida después de aplicar el instrumento a la muestra seleccionada

\begin{tabular}{|c|c|c|c|c|c|c|c|c|c|c|c|c|c|}
\hline Casos/indicadores & $\mathrm{P} 2$ & $\mathrm{P} 4$ & P5 & P6 & P9 & $\begin{array}{c}\mathrm{P} 1 \\
1\end{array}$ & $\begin{array}{c}\text { P1 } \\
2\end{array}$ & $\begin{array}{c}\text { P1 } \\
4\end{array}$ & $\begin{array}{c}\mathrm{P} 1 \\
5\end{array}$ & $\begin{array}{c}\mathrm{P} 1 \\
6\end{array}$ & $\begin{array}{c}\mathrm{P} 2 \\
2\end{array}$ & $\begin{array}{c}\mathrm{P} 2 \\
3\end{array}$ & $\begin{array}{c}\mathrm{P} 2 \\
4\end{array}$ \\
\hline 1 & 3 & 1 & 5 & 5 & 1 & 3 & 3 & 2 & 2 & 3 & 3 & 5 & 2 \\
\hline 2 & 2 & 2 & 4 & 4 & 1 & 2 & 1 & 2 & 2 & 2 & 2 & 2 & 2 \\
\hline 3 & 1 & 3 & 2 & 3 & 3 & 3 & 3 & 3 & 3 & 3 & 4 & 4 & 2 \\
\hline 4 & 2 & 4 & 1 & 2 & 2 & 2 & 2 & 2 & 2 & 3 & 2 & 3 & 3 \\
\hline 5 & 1 & 1 & 4 & 5 & 4 & 3 & 2 & 4 & 4 & 4 & 5 & 4 & 3 \\
\hline 6 & 4 & 1 & 2 & 3 & 1 & 1 & 2 & 2 & 2 & 3 & 3 & 2 & 1 \\
\hline 7 & 3 & 5 & 1 & 2 & 1 & 2 & 1 & 3 & 3 & 2 & 1 & 2 & 3 \\
\hline 8 & 3 & 5 & 3 & 2 & 1 & 1 & 1 & 4 & 2 & 3 & 3 & 5 & 5 \\
\hline 9 & 3 & 4 & 3 & 2 & 1 & 2 & 1 & 2 & 2 & 2 & 3 & 4 & 3 \\
\hline 10 & 1 & 5 & 2 & 1 & 3 & 3 & 4 & 1 & 1 & 1 & 1 & 1 & 1 \\
\hline 11 & 1 & 1 & 5 & 3 & 2 & 5 & 4 & 2 & 4 & 4 & 4 & 5 & 4 \\
\hline 12 & 2 & 5 & 2 & 1 & 4 & 2 & 1 & 2 & 2 & 2 & 3 & 4 & 2 \\
\hline 13 & 2 & 2 & 3 & 3 & 1 & 3 & 2 & 2 & 2 & 2 & 3 & 4 & 2 \\
\hline 14 & 1 & 4 & 3 & 1 & 5 & 5 & 5 & 1 & 2 & 3 & 3 & 4 & 3 \\
\hline 15 & 1 & 1 & 1 & 3 & 3 & 1 & 2 & 1 & 3 & 2 & 1 & 2 & 5 \\
\hline
\end{tabular}

Fuente: Elaboración propia.

Para caracterizar las dificultades lectoras de la muestra, se tomaron como estadísticos descriptores: la media $(\bar{x})$, mediana $(\hat{x})$, desviación estándar $(s)$ y cuartiles 1 y $3\left(Q_{1}\right.$ y $\left.Q_{3}\right)$. Para la estimación de los parámetros de la población, se utilizaron intervalos de confianza (IC) del 95\% para la media. La Tabla 5 resume los estadísticos obtenidos a partir de la matriz de datos.

Tabla 5

Resultados tabulares

\begin{tabular}{lcccccc}
\hline Variable & $\bar{x}$ & $I C(95 \%)$ & $\hat{x}$ & $S$ & $Q_{1}$ & $Q_{3}$ \\
\hline Estado visual & 2,2 & {$[2.02,2.34]$} & 2,0 & 1,07 & 1,0 & 3,0 \\
Memoria & 2,4 & {$[2.26,2.48]$} & 2,5 & 0,72 & 2,0 & 3,0 \\
Atención & 2,2 & {$[2.08,2.36]$} & 2,0 & 0,89 & 2,0 & 3,0 \\
Motivación & 2,8 & {$[2.77,2.97]$} & 3,0 & 0,65 & 2,5 & 3,3 \\
Comprensión & 2,3 & {$[2.24,2.39]$} & 2,3 & 0,51 & 2,0 & 2,6 \\
\hline
\end{tabular}

Fuente: Elaboración propia

A continuación, se presenta el Gráfico 1, de cajas y bigotes, que muestra los resultados obtenidos para cada una de las variables analizadas (estado visual, memoria, atención, motivación, y compresión). 


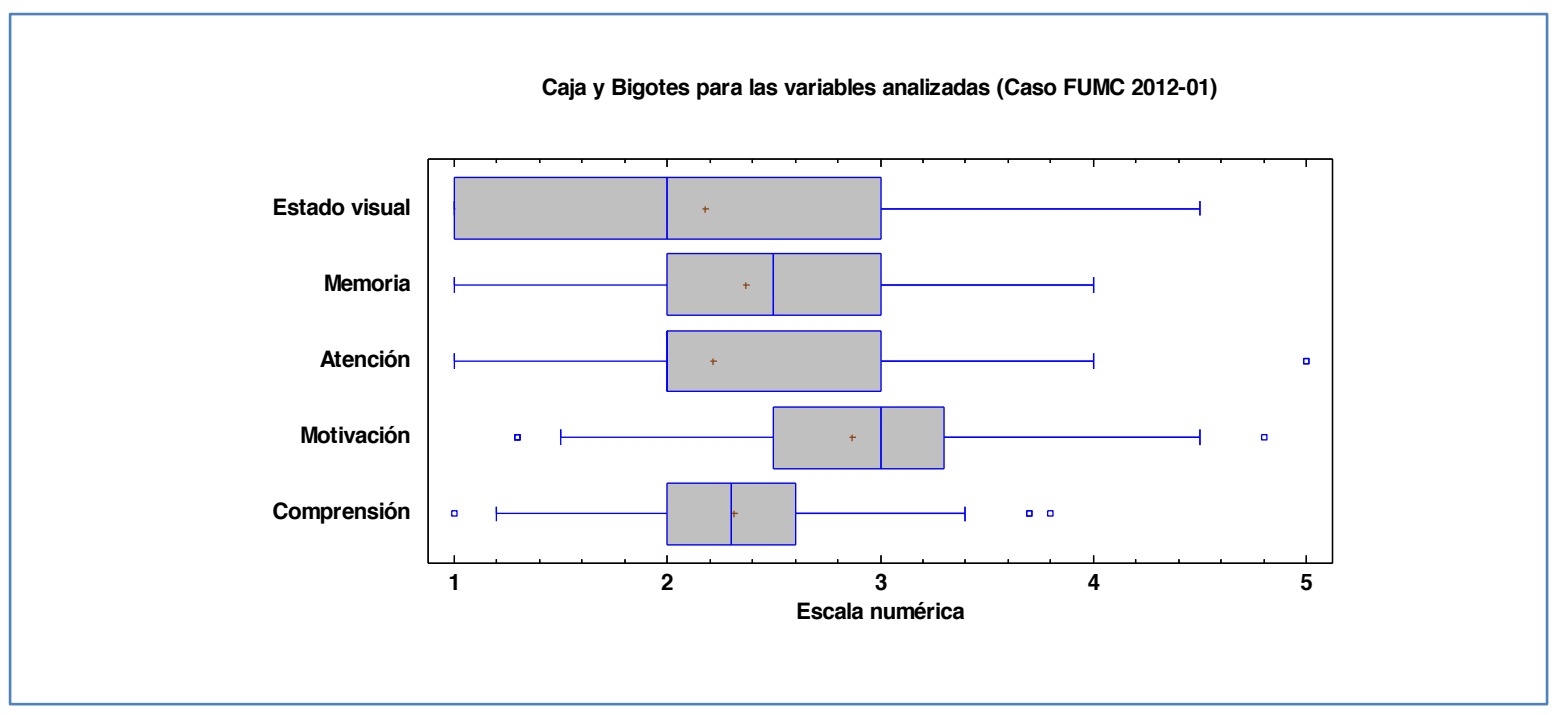

Gráfico 1. Cajas y bigotes para cada una de las variables. Fuente: Elaboración propia.

Se observa en el Gráfico 1, que el promedio del grupo de estudiantes evaluados para la variable Estado visual es inferior a 3.0 y que el $50 \%$ de ellos se encuentra en una puntuación inferior a 2.0; se visualiza también en la Tabla 5 que esta es la variable que presenta mayor dispersión ( $s=1.07$ ); además, el cuartil inferior $\left(Q_{1}\right)$ para esta variable es 1.0 y el superior $\left(Q_{3}\right)$ es 3.0, lo que indica que en general, los estudiantes al momento de ingresar a la FUMC, no consideran tener una buena visión y que en algunos casos no utilizan ayudas visuales; solo alrededor del $25 \%$ de ellos puntúa por encima de 3.0 en esta variable.

En lo referente a la variable Memoria, se observa que su promedio se encuentra por debajo de 3.0, en tanto que la calificación del $50 \%$ de los estudiantes en esta variable está por debajo de 2.5. También se evidencia en el gráfico que lo normal para los estudiantes que ingresaron en la cohorte analizada es que la puntuación para el estado de memoria a corto y largo plazo percibido se encuentra entre 2.0 y
3.0 (puntuaciones que corresponden a los cuartiles uno y tres).

En el Gráfico 1 se observa en la variable Atención que el promedio de la pregunta “¿Es atento al leer?" corresponde a 2.2; adicionalmente, el $50 \%$ de los participantes puntúa por debajo de 2.0 , el $25 \%$ califica por encima de 3.0 y hay un caso que se considera estadísticamente atípico y que puntúa en 5.0.

En el caso de la variable Motivación, puede observarse que el promedio es el mayor de todos (sin dejar de ser preocupante este resultado) y se ubica en 2.8. El 50\% de los estudiantes de la muestra puntúa por debajo de 3.0, además los cuartiles 1 y 3 se ubican en 2.5 y 3.3 respectivamente; es decir, solo el $25 \%$ de los estudiantes supera la puntuación de 3.3 en motivación para la lectura, considerándose estadísticamente atípico puntuaciones por encima de 4.0, para lo cual solo se presenta un caso.

En la variable Comprensión, puede establecerse a partir del Gráfico 1 que su promedio se ubica 
también por debajo de 3.0; el 75\% de los casos $\left(Q_{3}\right)$ puntúa por debajo de 2.6. Esta es la variable que presenta menor dispersión $(s=0.51)$, aunque se evidenciaron dos casos que se consideran estadísticamente atípicos, los que puntúan entre 3.6 y 4.0 .

\section{Discusión}

A partir de los resultados expuestos anteriormente, por un lado, se caracterizaron las dificultades lectoras percibidas por los estudiantes y, por el otro, se evidenció que los estudiantes del primer semestre 2012 de la Fundación Universitaria María Cano, perciben dificultades en cada una de las variables independientes analizadas (Estado visual, Memoria, Atención, Motivación y Comprensión). A continuación, se discuten los resultados de cada variable.

En el Estado visual, los estudiantes perciben que no tienen una buena visión y, en algunos casos, no utilizan ayudas visuales. Esta situación tiene implicaciones en la interpretación del mensaje escrito, pues al no tener normalidad en un órgano periférico como la vista o apoyos visuales como gafas u otros, los códigos no serán percibidos e interpretados adecuadamente. La percepción es el primer paso para que se inicie el proceso comunicativo entre autor y lector; si hay dificultades en esta función no podrá hablarse de efectividad comunicativa, lo cual trasciende el rendimiento lector.

Así mismo, puede evidenciarse a partir de los resultados estadísticos una percepción por parte de los estudiantes de dificultades en la Memoria. Es pertinente considerar que cuando existe una relación comunicativa entre el lector y el autor, las ideas que van surgiendo deben ser memorizadas para que exista comprensión global del texto. Cuando se perciben dificultades en la memoria, ello puede implicar que la información (mensaje) no logra ser comprendida, afectando la lógica cohesiva del discurso y, en consecuencia, la interpretación de las ideas principales y secundarias.

Otro aspecto relevante es la falencia percibida para almacenar la información leída a largo plazo; si esta percepción refleja una dificultad real implicaría que poco tiempo después de haber leído un texto la información es olvidada, generando vacíos conceptuales que inciden en el rendimiento lector $y$, por ende, en las funciones académicas.

En cuanto a la variable Motivación, puede decirse que los estudiantes del primer semestre a veces se sienten motivados, aunque en la investigación es evidente que los estudiantes evaluados no se perciben sintiendo interés personal o académico por la lectura, si dicha percepción corresponde a una actitud real, ello afectaría el resultado del proceso lector y las acciones que se deriven de este. Es necesario mencionar que los resultados obtenidos en esta variable causan interés como tema para otro estudio $y$ es pertinente explorar los motivos que inducen a una baja puntuación en la misma cuando se espera que, por encontrarse en el primer semestre, los estudiantes se sientan motivados y que las expectativas por el rendimiento personal y académico sean altas.

De manera análoga, se analizó la Atención, dispositivo de aprendizaje necesario en el proceso lector que está relacionado con la memoria; también 
se evidencia que los estudiantes perciben que al leer no están atentos, lo que puede repercutir negativamente en el procesamiento de la información textual, ocasionando vacíos conceptuales y omisión de información fundamental como la idea principal. Lo anterior puede incidir en el rendimiento lector, el manejo adecuado del tiempo y el interés por la actividad lectora, entre otros.

Finalmente, los resultados de la variable Comprensión, indican estadísticamente que hay una percepción de dificultades en el proceso lector para: leer en silencio y en voz alta, identificar ideas principales y secundarias, en la comprensión del mensaje escrito, en la utilización de vocabulario amplio que incluye sinónimos y antónimos, en el uso de signos de puntuación y en la identificación de errores ortográficos. La percepción de los problemas descritos anteriormente y si estos se manifiestan en la realidad revelaría, en primer lugar, que los estudiantes posiblemente egresaron de secundaria con falencias en los elementos evaluados; en segundo lugar, que no han tenido interés personal por mejorar las deficiencias en la lectura; y por último, que la compresión de los mensajes escritos es limitada, lo que significa que no hay decodificación-compresión, los cuales son procesos que afectan el rendimiento lector y la comunicación entre emisor y perceptor.

En síntesis, los estudiantes de primer semestre en esta universidad manifiestan percibir las dificultades que podrían afectar a las distintas variables implicadas en el proceso lector. Sería, por lo tanto, muy interesante corroborar esta percepción evaluando directamente su lectura.

Al respecto, es pertinente mencionar que uno de los limitantes para el desarrollo de esta investigación fue la carencia de un instrumento (prueba) para evaluar la lectura, puesto que si bien existen pruebas a nivel internacional, estas no se encuentran validadas en la población colombiana y las que existen en Colombia son para niños y adolescentes, y no para la población universitaria. Esta limitación permitió elaborar una reflexión académica con relación al rol que el fonoaudiólogo realiza en la evaluación del proceso lector como parte fundamental del lenguaje; por lo tanto, se generó un nuevo proyecto que consiste en el diseño de un instrumento válido y confiable para evaluar la comprensión lectora, el lenguaje, la atención y la memoria operativa para estudiantes universitarios.

Este instrumento se convertirá en una herramienta básica para ser utilizada por los fonoaudiólogos de este país que tengan relación con el sector educativo y que estén en función del fortalecimiento académico y del interés en evitar la deserción escolar, los cuales son temas de preocupación en el contexto nacional. 


\section{Referencias}

Abusamra, V., Cartoceti, R., Raiter, A., y Ferreres, A. (2008). Una perspectiva cognitiva en el estudio de la comprensión de textos. Psico, 39(3), 352-361.

Bravo, L. (2004). Las destrezas perceptuales y los retos en el aprendizaje de la lectura y la escritura. Una guía para la exploración y comprensión de dificultades específicas. Revista Electrónica Actualidades Investigativas en Educación, 4(1), 24.

Carpenter, P., Miyake, A., \& Just, M. (1994). Working memory constraints in comprehension: Evidence from individual differences, aphasia, and aging. San Diego, CA: Academic Press.

Defior, S. (1993). Las dificultades de lectura: papel que juegan las diferencias de lenguaje. Comunicación, Lenguaje y Educación, 17, 3-14.

Díaz, J., Martínez, I., y Ávila, Y. (2006). Percepción cognitiva de los profesores sobre la motivación lectora de los alumnos. Revista Latinoamericana de Estudios Educativos, 36, 3-4.

Duque, C., Vera, A., y Hernández, A. (2010). Comprensión inferencial de textos narrativos en primeros lectores: una revisión de la literatura. Revista OCNOS, 6, 35-44.

Ellery, V. (2005). Un aula de lectura y escritura comprehensiva e integradora. Lectura y Vida Revista Latinoamericana de Lectura, 26(1), 38-51.

Etchepareborda, M. C. y Abad-Mas, L. (2005). Memoria de trabajo en los procesos básicos del aprendizaje. Revista de Neurología, 40(1), 79-83.

León, J. (2010). Neuroimagen de los procesos de comprensión en la lectura y el lenguaje. Revista Nebrija de Lingüística Aplicada a la Enseñanza de Lenguas, 7, 61-71.

Logie, R. H. (1995). Visuo-spatial Working Memory. Hove, UK: Lawrence Erlbaum.

López, A. y Guevara, Y. (2008). Programa para prevención de problemas en la adquisición de la lectura y la escritura. Revista Mexicana de Análisis de la Conducta, 1, 57-78.
Muñoz-Valenzuela, C. y Schesltraete, M. A. (2008). Decodificación y comprensión de lectura en la edad adulta: ¿una relación que persiste? Revista Iberoamericana de Educación, 45(5), 6.

Preilowski, B. y Matute, E. (2011). Diagnóstico neuropsicológico y terapia del Trastorno de LecturaEscritura (dislexia del desarrollo). Revista Neuropsicología, Neuropsiquiatría y Neurociencias, 11(1), 95-122.

Suárez, A., Moreno, J., y Godoy, M. (2010). Vocabulario y comprensión lectora: algo más que causa y efecto. Revista de la Red de Universidades Lectoras, 1. http://dx.doi.org/10.15645/Alabe.2010.1.7

Villamizar, G. (2003). El lenguaje en la comprensión de la lectura. Acción Pedagógica, 12(2), 86-94.

Zorrilla, M. J. P. (2005). Evaluación de la comprensión lectora: dificultades y limitaciones. Sociedad Lectora y Educación, Número extraordinario, 121-138. 